\title{
PNA, antisense, and antimicrobials
}

\author{
David J. Ecker and Susan M. Freier
}

A key objective for those interested in the discovery of new antimicrobial agents is the determination of the genes essential for survival of pathogenic organisms. Novel microbial genes and their function have been the focus of intense research over the past two decades, and the publication and analysis of an ever-increasing number of microbial genomes is testament to the power of modern sequencing techniques. Clearly, sequencing has advanced faster than our ability to analyze and understand gene function. To keep up, new techniques and tools are needed to enable high-throughput functional analysis. In this issue, Liam Good and Peter Nielsen ${ }^{1}$ demonstrate one such tool using antisense peptide nucleic acid (PNA) to specifically inhibit microbial gene function with potential use for target validation in bacteria.

PNA (which, strictly speaking, is neither peptide nor acid) is a molecule of synthetic origin that binds to single-stranded nucleic acids. Instead of a repeating sugar-phosphate backbone, it has a repeating polyamide structure to which the purine and pyrimidine bases are attached in a fashion that confers the ability to bind RNA by Watson-Crick hybridization. The unnatural chemical structure of PNA provides stability in cells because neither nucleases nor peptidases will cleave it.

In their paper, Good and Nielsen' demonstrate that PNA can inhibit gene expression with compelling specificity both in cell extracts and in whole bacteria. Two different bacterial RNAs were targeted with complementary PNAs and a randomized control sequence. The complementary PNAs inhibited their intended target, whereas the other PNAs did not. To further demonstrate specificity, the sequence of one of the RNA target sites was changed by introduction of silent mutations into host DNA. Two mutations resulted in a significant loss of activity and six mutations completely eliminated PNA activity. When the PNA was "corrected" to match the mutant RNA, PNA activity was fully restored, whereas the original wild-type PNA was inactive.

These results provide a hint of the potential of PNA (or one of its chemically modi-

David J. Ecker is vice president of combinatorial drug discovery (decker@isisph.com) and Susan $M$. Freier is director of molecular and structural biology (sfreier@isisph.com) at Isis Pharmaceuticals, Carlsbad Research Center, 2292 Araday Ave., Carlsbad, CA 92008.

Table 1. Comparison of antisense to genetic knockout for validation of bacterial targets.

PNA antisense

Breadth of

applicability

Any bacterial species. May require development of delivery conditions for each species

$\begin{array}{lll}\text { Sensitivity Level of inhibition can be regulated. } & \text { Usual effect on expression is all } \\ \text { Time course of inhibition can be controlled } & \begin{array}{l}\text { or none }\end{array}\end{array}$

"Leakiness" Difficult to knock out $100 \%$ of target, but reduced levels of target activity may more realistically mimic the effect of a therapeutic inhibitor

fied derivatives) as an antimicrobial agent. If it were possible to design agents around PNA, it would be a significant breakthrough in antimicrobial drug discovery: Virtually any microbial gene could be targeted and highly organism-specific drugs could be envisioned. As with any antisense drug, the sequence could be changed to stay ahead of drug resistance mediated by the bacteria mutating the drug target site. Bacteria themselves use antisense as a natural mechanism to inhibit specific gene expression ${ }^{2,3}$, and the present work demonstrates that PNAs inside bacteria are effective and specific inhibitors of their target genes.

The obvious caveat, however, is bacterial cell membrane penetration. Indeed, Good and Nielsen found that wild-type bacteria were barely affected by antisense PNA; however, a bacterial strain with a defect in its membrane could be inhibited at low micromolar concentrations of antisense PNA, suggesting that bacterial cell uptake was limiting. Surprisingly, very little is known about why some rather large molecules penetrate bacterial cells while others do not ${ }^{4}$. For example, the macrolide and aminoglycoside antibiotic families are relatively large molecules that enter bacterial cells and bind to RNA targets inside the cell. Exactly how they enter into the cell is a mystery.

Whether antisense PNAs could be used directly as antimicrobial agents needs to be addressed with more experiments. There have been several reported efforts to use antisense oligonucleotide analogs as antibacterial agent $s^{5-9}$, but these have achieved only mixed results. PNA is chemically different from previously tested oligonucleotide analogs in that the backbone is uncharged. The impact of such a major change on membrane transport issues should be significant. If uptake is the limiting factor, it is possible that advances in formulation or further chemical modification of PNA could increase bacterial cell pen-

\section{Genetic knockout}

Effective in many bacteria, but some may not be amenable to genetic manipulation (e.g., Mycobacterium tuberculosis)

$100 \%$ of target usually knocked out. Secondary mutations or physiological compensation can mask knockout phenotype

etration. Chemical modification and formulation of oligonucleotides has resulted in successful delivery of oligonucleotides ${ }^{10,11}$. Similar success should be possible for delivery of PNAs to bacteria.

In contrast to therapeutic applications, which will require considerable further development, antisense PNA for target validation is more immediately applicable. The delivery of PNA to bacteria in the laboratory is a much more tractable problem than in the clinic. A variety of strategies exist to deliver compounds to bacterial cells in the laboratory, including electroporation, permeabilizing solvents, cationic lipid formulations, and pore-forming peptides. Exactly what will work for PNA remains to be determined.

The current technology for measuring the quality of a candidate gene as a good drug discovery target is based upon well-established genetic knockout methods, which are now being accelerated with the use of highthroughput instrumentation. Table $1 \mathrm{com}$ pares PNA antisense to genetic knockout for validation of bacterial targets. From this table, it is clear that both techniques are useful and synergistic: Antisense for bacteria is likely to make very good sense for the pharmaceutical industry in target validation.

\footnotetext{
1. Good., L. and Nielsen, P.E. 1998. Nature Biotechnology 16:355-358.

2. Wagner, E.G.H. and Simons, R.W. 1994. Annu. Rev. Microbiol. 48:713-742.

3. Eguchi, Y., Itoh, T., and Tomizawa, J. 1991. Annu. Rev. Biochem. 60:631-652.

4. Nikaido, H. 1994. Science 264:382-388.

5. Gasparro, F.P. et al. 1991. Antisense Res. Dev. 1:117-140.

6. Rahman, M.A. et al. 1991. Antisense Res. Dev. 1:319-327.

7. Lupski, J.R. and Katz, L. 1990. WO 900624A1.

8. Chrisey, L.A. et al. 1993. Antisense Res. Dev. 3:367-381.

9. Rapaport, E. et al 1996. Proc. Natl. Acad. Sci. USA 93:709-713.

10. Bennett, C. F. et al. 1996. J. Control. Rel. 41:121-130.

11. Litzinger, D.C. et al. 1996. Biochim. Biophys. Acta 1281:139-149.
} 